\title{
Basic symptoms in early psychotic and depressive
}

\section{disorders}

\author{
FR AUKE SCHULTZE-LUTTER, STEPHAN RUHRMANN, HEINZ PICKER, \\ HEINRICH GRAF VON REVENTLOW, ANKE BROCKHAUS-DUMKE \\ and JOACHIM KLOSTERKÖTTER
}

\section{Background Depression is a frequent condition in early psychosis. Therefore, early detection instruments should distinguish depression from beginning psychosis.}

\begin{abstract}
Aims To examine whether basic symptoms, i.e. subtle subjective deficits, differ between participants suffering from a potential prodrome $(n=146)$, firstepisode schizophrenia $(n=153)$ and nonpsychotic depression $(n=|| 5)$.
\end{abstract}

\begin{abstract}
Method Basic symptoms were assessed with the Schizophrenia Proneness Instrument.
\end{abstract}

\section{Results The prodrome and} schizophrenia groups did not differ in level of basic symptoms but both had higher levels than the depression group. DSM-IV depression was frequent in those suffering from a potential prodrome (38\%) and first-episode schizophrenia (21\%). In both groups, participants with and without depression did not differ in basic symptoms. In multivariate analyses, consideration of current depression generally facilitated correct group classification, except for participants suffering from both a potential prodrome and depression.

\section{Conclusions Cognitive basic}

symptoms distinguished well between all three groups. However, identification of persons suffering from a potential prodrome might be enhanced by considering current affective status.

Declaration of interest None. Funding detailed in Acknowledgements.
Clinically relevant depression is frequent in first-episode schizophrenia and its early, prodromal states (for example Koreen et al, 1993; Rosen et al, 2006). Furthermore, depressive symptoms have been suggested to frequently mark the onset of the initial prodrome of psychosis (Häfner et al, 1999). Thus, an instrument for the early detection of psychosis has to distinguish potentially prodromal individuals from individuals suffering primarily from a depressive disorder.

Basic symptoms, especially subtle, subjective disturbances of thought, speech and perception, as assessed with the Bonn Scale for the Assessment of Basic Symptoms (BSABS; Gross et al, 1987; Huber \& Gross, 1989), were shown to be predictive of later schizophrenia (Klosterkötter et al, 2001) and to distinguish between non-psychotic affective disorders and schizophrenia (Klosterkötter et al, 1996). However, a direct comparison between patients with non-psychotic unipolar depressive disorder, those with potential prodrome and those suffering from a first-episode schizophrenia has not been studied.

\section{METHOD}

\section{Inclusion and exclusion criteria}

The initial prodrome of psychosis was defined by the presence of at least any one cognitive-perceptive basic symptom found predictive for the development of schizophrenia in the Cologne Early Recognition study (Klosterkötter et al, 2001) with first occurrence at least $\mathbf{1 2}$ months earlier and multiple occurrences within 1 of the past 3 months, i.e. a minimum rating of ' 3 ' on the Schizophrenia Proneness Instrument. These include thought interferences, perseveration, pressure or blockages; disturbances of receptive language, decreased ability to discriminate between ideas and perception or fantasy and true memories, unstable ideas of reference, derealisation; and visual or acoustic perception disturbances. Participants were between 16 and 40 years. Presence of any ultra-high risk criterion (Phillips et al, 2000) served neither as an intake nor as an exclusion criterion.

Inclusion criterion for the schizophrenia group was a first episode of schizophrenia according to DSM-IV criteria (American Psychiatric Association, 1994), and for the depression group an episode of major depressive disorder, dysthymic disorder or depressive disorder not otherwise specified according to DSM-IV without psychotic features for which help was sought for the first time.

General exclusion criteria were: (a) diagnosis of delirium, dementia, amnestic or other cognitive disorders, mental retardation, psychiatric disorders due to a somatic factor or related to psychotropic substances according to DSM-IV, (b) alcohol or drug abuse within the past 3 months according to DSM-IV and (c) diseases of the central nervous system (inflammatory, traumatic, epilepsy). In addition, current or past diagnosis of any psychotic disorder according to DSM-IV criteria served as an exclusion criterion in the prodromal and depression group. In the schizophrenia group, the latter was restricted to a past diagnosis of a psychotic disorder. An exception to this in the prodromal and schizophrenia group was psychosis not otherwise specified, when rated due to the presence of brief limited intermittent psychotic symptoms as defined by the ultra-high risk criteria (Phillips $e t a l$, 2000). The presence of attenuated or brief limited intermittent psychotic symptoms according to the ultra-high risk criteria was a further exclusion criterion in the depression group. In the matter of basic symptoms in the depression group, only the 10 cognitive-perceptive basic symptoms relevant for inclusion into the prodromal group were limited to not exceeding an occurrence of once in a month within the past 3 months, i.e. a maximum rating of ' 2 ' on the Schizophrenia Proneness Instrument.

\section{Participants}

Of the 414 participants included in the study, 146 were suffering from a potential prodrome, 153 from first-episode schizophrenia $(70 \%$ paranoid, $20 \%$ undifferentiated, $9 \%$ disorganised and $1 \%$ catatonic subtype) and 115 from depression (84\% major depressive disorder, with $73 \%$ melancholic, $16 \%$ atypical and $11 \%$ no 
melancholic, atypical or catatonic features; $2 \%$ dysthymic disorders, equally with and without major depressive episode; and $14 \%$ depressive disorders not otherwise specified, with $69 \%$ recurrent brief depressive disorder and $31 \%$ minor depressive disorder). All gave written informed consent to the study between June 2000 and December 2005, with recruitment of participants suffering from a potential prodrome ending in December 2003. The majority of them had sought help at the Cologne Early Recognition and Intervention Centre for mental crisis (FETZ); others, especially those with first-episode schizophrenia, were in-patients of the Department of Psychiatry and Psychotherapy of the University of Cologne. In addition to the inclusion criteria, $80 \%$ of participants suffering from a potential prodrome reported attenuated psychotic symptoms, and only 1 person $(0.7 \%)$ supplementary brief limited intermittent psychotic symptoms. Group differences for all socio-demographic characteristics are presented in Table 1.

Age was significantly correlated with graduation (Spearman's $r_{\mathrm{S}}=0.316, \quad P=$ $0.000)$, vocational education $\left(r_{\mathrm{S}}=0.456\right.$, $P=0.000)$ and partnership $\left(r_{\mathrm{S}}=0.232\right.$, $P=0.000$ ), which was also positively correlated with female gender $\left(r_{\mathrm{S}}=0.397\right.$, $P=0.000)$. Graduation and vocational education were weakly associated to each other $\left(r_{\mathrm{S}}=0.197, P=0.000\right)$ and to current occupation $\quad\left(r_{\mathrm{S}}=0.208 / 0.188, \quad\right.$ each $P=0.000$ ).

\section{Instruments}

The subtle, self-experienced, self-reported deficits that often remain solely in the self-perception of the patient and do not show in behaviour, i.e. basic symptoms, were assessed with the Schizophrenia Proneness Instrument, Adult version (SPI-A; Schultze-Lutter et al, 2007). The 34-item SPI-A was derived from BSABS data on 79 individuals with a true prodrome and 346 with remitted schizophrenia, by cluster and confirmatory facet analyses for use by experienced and trained clinicians. Being a semi-structured interview, the SPI-A was designed to be applied together with the Structured Interview for Prodromal Syndromes (SIPS; Miller et al, 2002), assessing attenuated and brief limited intermittent psychotic symptoms, and the Positive And Negative Syndrome Scale (PANSS; Kay et al, 1987) to cover the whole range from early prodromal basic symptoms via attenuated and brief limited intermittent to frank psychotic symptoms. The SPI-A comprises 6 sub-scales of 5 to 6 items each:

(1) affective-dynamic disturbances, including an impaired tolerance to certain stressors, a change in general mood and a decrease in emotional responsiveness in general as well as towards significant others or special events

(2) cognitive-attentional impediments, including some of the less specific cognitive basic symptoms (Klosterkötter et al, 2001), i.e. an inability to divide attention between tasks relying on different senses, for example between

talking and preparing a sandwich, feeling overly distracted by all kinds of stimuli, difficulties with short-term memory and concentration as well as slowed-down thinking and lack of purposive thoughts

(3) cognitive disturbances comprising the more specific cognitive basic symptoms (Klosterkötter et al, 2001), i.e. an increased indecisiveness with regard to making minor decisions or choices, disturbances of immediate recall within seconds, interference of emotionally and otherwise insignificant thoughts, thought blockages and disturbances of receptive as well as expressive speech

(4) disturbances in experiencing self and surroundings, including self-reported

Table I Characteristics of sample

\begin{tabular}{|c|c|c|c|c|}
\hline & $\begin{array}{l}\text { Potential pro- } \\
\text { drome group } \\
\quad(n=\mid 46)\end{array}$ & $\begin{array}{l}\text { First-episode } \\
\text { schizophrenia } \\
\text { group } \\
(n=153)\end{array}$ & $\begin{array}{c}\text { Unipolar } \\
\text { depression } \\
\text { group } \\
(n=I 15)\end{array}$ & $\begin{array}{c}P \\
\text { All groups' } \\
\text { (post-hoc) }^{2}\end{array}$ \\
\hline Age, mean ( \pm s.d.) & $24.4( \pm 5.2)$ & $26.7( \pm 6.5)$ & $27.6( \pm 7.7)$ & 0.000 \\
\hline median (range) & $24(16-39)$ & $25(17-43)$ & $26(17-52)$ & $(0.00 \mathrm{I} / 0.000 / \mathrm{NS})$ \\
\hline \multirow[t]{2}{*}{ Gender, \% male } & 69.2 & 74.5 & 47.0 & 0.000 \\
\hline & & & & (NS/0.000/0.000) \\
\hline \multicolumn{5}{|l|}{ Partnership, \% } \\
\hline Single & 61.4 & 77.1 & 62.7 & 0.031 \\
\hline Married/steady partner & 37.2 & 22.2 & 35.5 & $(0.013 / \mathrm{NS} / 0.037)$ \\
\hline Separated & 1.4 & 0.7 & 1.8 & \\
\hline \multicolumn{5}{|l|}{ Graduation ${ }^{3}, \%$} \\
\hline None & 2.8 & 3.3 & 0.9 & $<0.001$ \\
\hline CSE (10 years) & 5.5 & 23.3 & 13.2 & $(0.000 / N S / N S)$ \\
\hline O-level (10 years) & 14.5 & 20.5 & 20.2 & \\
\hline VBD $(12$ years $)$ & 13.1 & 9.9 & 9.6 & \\
\hline A-level (13 years) & 49.7 & 39.7 & 48.2 & \\
\hline Still in school & 14.5 & 3.3 & 7.9 & \\
\hline \multicolumn{5}{|l|}{ Vocational education, \% } \\
\hline None & 13.1 & 29.4 & 15.9 & 0.001 \\
\hline Apprenticeship or similar & 15.2 & 28.1 & 27.4 & $(0.000 / \mathrm{NS} / 0.036)$ \\
\hline Master craftsman or similar & 1.4 & 2.0 & 4.4 & \\
\hline College of higher education & 2.8 & 1.3 & 2.7 & \\
\hline University & 9.7 & 4.6 & 11.5 & \\
\hline Still in school/training & 57.9 & 34.6 & 38.1 & \\
\hline \multicolumn{5}{|l|}{ Current occupation, \% } \\
\hline None & 16.2 & 36.6 & 20.5 & 0.001 \\
\hline Therapeutic/sheltered & - & 1.3 & 0.9 & $(0.000 / N S / 0.036)$ \\
\hline Regular including education & 83.1 & 59.9 & 75.9 & \\
\hline Other & 0.7 & 1.3 & 2.7 & \\
\hline
\end{tabular}

CSE, Certificate of Secondary Education; VBD, Vocational baccalaureate diploma

NS: $P \geqslant 0.05$

I. F-test or $3 \times \mathrm{k}-\chi^{2}$-test.

2. Unadjusted $t$-tests or $2 \times \mathrm{k}$ - $\chi$-tests (prodrome $v$. schizophrenia/prodrome $v$. depression/schizophrenia $v$. depression).

3. Translated into British graduations (years of school education required to receive the respective graduation). 
pressure of unrelated thoughts, unstable ideas of reference, a self-recognised disturbance in the visual perception of others, a decreased capacity to distinguish between different kinds of emotions and an increased emotional reactivity in response to routine social interactions

(5) body perception disturbances comprising various kinds of coenesthetic phenomena

(6) perception disturbances consisting of hypersensitivity to light/optic stimuli and/or to sounds/noise, photopsia, micro-/macropsia, changes in the perception of the intensity/quality of acoustic stimuli and somatopsychic bodily depersonalisation, all of these clearly distinct from psychotic experiences by being related to a malfunction of the individual's own senses or mind.

For the quantitative rating, a 7-point severity scale was introduced with maximum frequency of occurrence within the past 3 months as the guiding criterion; i.e. from ' 0 ' $=$ 'symptom absent' to ' 6 ' $=$ 'present daily'. The overall concordance rate of the four SPI-A interviewers with an expert rating (F.S.-L.) was $91 \%$.

The Structured Clinical Interview for DSM-IV (SKID-I, German version; Wittchen et al, 1997) was conducted to rule out past or present psychotic disorders and confirm diagnoses in the schizophrenia and depression group as well as to assess comorbidity with affective disorders in the schizophrenia and potential prodrome group.

\section{Data analysis}

SPI-A sub-scale totals at baseline were compared between groups by two-tailed Mann-Whitney tests and adjusted for multiple testing across both, the pairwise group comparisons and sub-scales (18 pairwise tests in all), according to the sequential method for ordinal data by Holm (1979).

Despite the socio-demographic group differences, no covariance analyses were carried out, as nonparametric correlation analyses of the whole sample had only revealed weak, negligible associations between the socio-demographic and psychopathological data, these being highest for age and affective-dynamic disturbances $\left(r_{\mathrm{S}}=0.142, P=0.004\right)$ followed by associations between age and perception disturbances $\left(r_{S}=0.108, P=0.028\right)$ and gender and affective-dynamic disturbances $\left(r_{\mathrm{S}}=\right.$ $0.108, P=0.029$ ).
For evaluation of the combined ability of the SPI-A sub-scales to discriminate participants with depression from those suffering from a potential prodrome as well as from first-episode schizophrenia and the influence of the main socio-demographic characteristics, age and gender, as well as the presence and kind of current depressive episode, stepwise logistic models (Waldmethod, forward selection, without inclusion of a constant term) were used on the depression and potential prodrome group (a) with intake of current depressive disorder and (b) without its consideration. To assess the predictive accuracy of the derived model in an unbiased manner, data-sets of the depression and potential prodrome group were randomly split into a model generation sample (MG; $n=132$ ) and a model validation sample (MV; $n=129$ ) with participants with schizophrenia taken into account as a further validation group expected to be classified as 'prodromal'.

To estimate the impact of a possible selection bias affecting the comparison between participants suffering from a potential prodrome and those with depression, additional subgroup comparisons were made. Such a selection bias might have been introduced by the inclusion criteria, of which about half of the items of cognitive disturbances ( 3 of 6 items), of disturbances in experiencing self and surroundings ( 3 of 5 items) and of perception disturbances ( 3 of 6 items) were - at a severity of at least ' 3 ' - part of the inclusion criteria of the prodrome. To assume that such a selection bias had not influenced the results, the following conditions, which centre on the schizophrenia group as the group included independently from any prerequisite regarding basic symptoms had to be met. If participants with schizophrenia but without current depression do not differ from either participants suffering from a potential prodrome without or with depression, and both potential prodrome subgroups do not differ from each other, then it can be concluded that the performance on the SPI-A of participants suffering from a potential prodrome is independent from the presence of current depression and not solely due to inclusion criteria, in which case it would be expected to exceed that of participants with schizophrenia but no depression. Furthermore, if schizophrenia subgroups with and without current depression show equal SPI-A subscale totals and the schizophrenia subgroup with depression outperforms the depression group but not the prodrome subgroup with depression (which again is assumed not to differ from the prodrome subgroup without depression) do not, then higher sub-scale totals of those suffering from a potential prodrome and depression as well as participants suffering from a potential prodrome but no depression in comparison to participants with depression alone can be assumed to reflect not solely the inclusion criteria but also 'true' group differences. Comparisons of subgroups were carried out by two-tailed Mann-Whitney tests and adjusted for multiple testing across sub-scales (6 pairwise tests each), according to Holm's method (Holm, 1979).

\section{RESULTS}

\section{Main group comparisons}

As expected, participants suffering from a potential prodrome generally reported as many and as severe basic symptoms at all levels as participants with schizophrenia (Fig. 1); only with regard to affectivedynamic disturbances and disturbances in experiencing self and surroundings, did the participants with schizophrenia show even higher mean totals than participants suffering from a potential prodrome (20.3 \pm 7.6 vs. $17.6 \pm 6.8, P=0.006$, and $12.3 \pm 6.3$ v. $10.1 \pm 5.3, P=0.009)$. Compared to participants with depression, significantly higher mean totals in the potential prodrome and schizophrenia groups were found for all SPI-A sub-scales (Fig. 1).

\section{Discrimination of depression group}

Fifty-six participants suffering from a potential prodrome $(38 \%)$ fulfilled criteria for a current depressive disorder: $34 \%$ of these had a current major depressive disorder, $11 \%$ a dysthymic disorder and $55 \%$ a depressive disorder not otherwise specified. Out of the major depressive disorders, $47 \%$ were of the melancholic, $16 \%$ of the atypical and $37 \%$ of the no melancholic, atypical or catatonic subtype. Out of the dysthymic disorders, $33 \%$ were accompanied by a major depressive episode. $71 \%$ of the depressive disorders not otherwise specified were recurrent brief depressive disorders and $29 \%$ minor depressive disorders. Participants suffering from a potential prodrome and depression and those suffering from a potential prodrome but no 


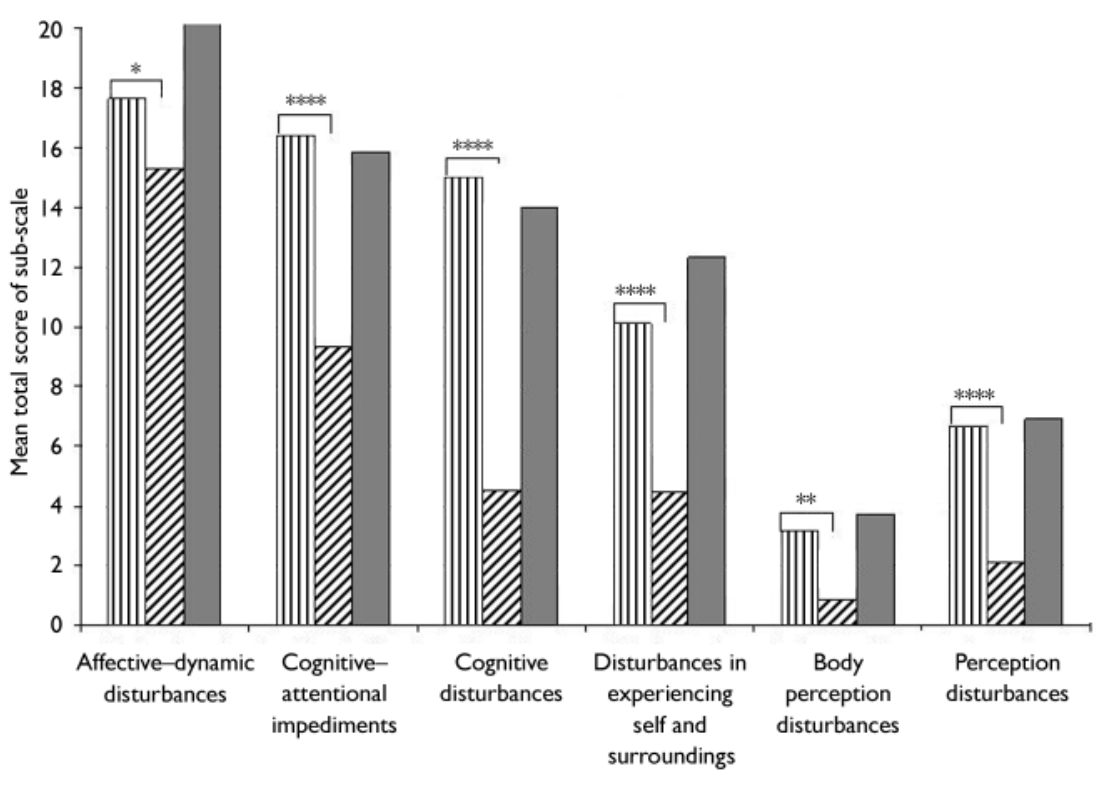

Fig. I Comparison of group means of the SPI-A sub-scale totals between participants suffering from a potential prodrome, [I]| first-episode schizophrenia, $\square$ and non-psychotic depression patients, $/ /$. MannWhitney Tests; $P$-values are adjusted for multiple testing across sub-scales and groups according to Holm's (1979) sequential method. $* P<0.05$; ** $P<0.0005$; *** $P<0.000000001$. All differences between participants with schizophrenia and those with depression are highly significant $(P<0.000 \mathrm{I})$.

depression $(n=90)$ did not differ in their socio-demographic characteristics, except slightly for partnership, with a tendency of those with additional depression to more frequently have a steady partner/spouse $\left(50 \% \quad v .30 \%, 2 \times 3 \quad \chi^{2}\right.$-test, d.f. $=2$, $P=0.046)$. Furthermore, the two subgroups did not differ in the percentage of persons with attenuated or brief limited intermittent psychotic symptoms.

Of the participants with first-episode schizophrenia, $32(21 \%)$ fulfilled criteria of a depressive disorder not otherwise specified: $47 \%$ of a recurrent brief depressive disorder, $31 \%$ of a major depressive disorder in addition to a psychotic disorder and $22 \%$ of a post-psychotic depressive disorder. They showed no difference in mean age to those with no current depression. There was a higher proportion of female subjects in the schizophrenia with depression group $\left(41 \% v .22 \%, 2 \times 2 \chi^{2}\right.$-test, d.f. $=1$, $P=0.027)$. Despite no difference in graduation and vocational education, participants with both schizophrenia and depression more frequently had a regular occupation than those with solely schizophrenia $(81 \%$ v. $54 \%, 2 \times 4 \chi^{2}$-test, d.f. $=3, P=0.019$ ). The greatest difference between participants with schizophrenia with and without depression was in the distribution of schizophrenia subtypes $\left(2 \times 4 \chi^{2}\right.$-test, d.f. $=3$,
$P=0.014)$ : participants with schizophrenia and depression were more frequently of the undifferentiated $(42 \% v .14 \%)$ and less frequently of the paranoid subtype $(52 \% v$. $75 \%)$.

Considering all six SPI-A sub-scales, age, gender and current depressive episode (i.e. major depressive, dysthymic or other depressive episode), a model, reg1 $\left(\chi^{2}=117.5\right.$, d.f. $\left.=4, P=0.000\right)$, was generated, which included cognitive disturbances $(\beta=0.203$, s.e $=0.069, \exp (\beta)=1.225$ with 95\% CI (1.070/1.403)), disturbances in experiencing self and surroundings $(\beta=0.170$, s.e. $=0.082, \exp (\beta)=1.186$ with $95 \%$ CI (1.009/1.394)), current major depressive episode $(\beta=-4.579$, s.e. $=0.782, \exp (\beta)=$ 0.010 with $95 \%$ CI $(0.002 / 0.048))$ and current episode of depression not otherwise specified $(\beta=-2.003$, s.e. $=0.714, \exp (\beta)=$ 0.135 with $95 \%$ CI $(0.003 / 0.547))$, classifying $89.4 \%$ of the participants with depression and those suffering from a potential prodrome of the model generation (MG) group, $93.8 \%$ of the depressionpotential prodrome model validation (MV) group and $96.1 \%$ of the participants with schizophrenia correctly (Table 2). In both, the potential prodrome and schizophrenia groups, misclassifications were due solely to subjects with current depression, thus leading to lower correct classification rates $(76.8$ and $81.3 \%$ ) in these respective subgroups (Table 2).

Taking current depression from the equation, a model, reg2 $\left(\chi^{2}=84.9\right.$, d.f. $=5$, $P=0.000$ ), including five parameters was developed: cognitive-attentional impediments $(\beta=-0.134$, s.e. $=0.057, \exp (\beta)=$ 0.875 with $95 \% \mathrm{CI}(0.782 / 0.979))$, cognitive disturbances $(\beta=0.322$, s.e. $=0.075, \exp (\beta)$ $=1.381$ with $95 \%$ CI $(1.191 / 1.600))$, disturbances in experiencing self and surroundings $(\beta=0.233$, s.e. $=0.076, \exp (\beta)=1.263$ with $95 \%$ CI $(1.089 / 1.465))$, age $(\beta=-0.069$, s.e. $=0.023, \exp (\beta)=0.933$ with $95 \%$ CI $(0.893 / 0.975))$ and gender $(\beta=-1.411$, s.e. $=0.549, \exp (\beta)=0.244$ with $95 \%$ CI $(0.083 / 0.715))$, correctly classifying $80.3 \%$ of the MG sample, $83.7 \%$ of the MV sample and $78.4 \%$ of participants with schizophrenia as well as $82.1 \%$ of the potential prodrome subgroup with depression and $75.0 \%$ of the schizophrenia subgroup with depression (Table 2).

\section{Group comparisons, evaluation of the impact of a selection bias}

Participants with schizophrenia but no depression did not differ from those suffering from a potential prodrome and depression but did differ from those suffering from a potential prodrome but no depression on affective-dynamic disturbances $\left(P_{\text {adjusted }}=0.0009\right)$ and disturbances in experiencing self and surroundings $\left(P_{\text {adjusted }}=0.0033\right.$; Fig. 2). Thereby, participants with schizophrenia but no depression had higher mean totals than those suffering from a potential prodrome but no depression, which was mainly due to higher mean ratings on the 3 items of a reduced stress tolerance in affective-dynamic disturbances (all $P_{\text {adjusted }}<0.0001$ ) and on unstable ideas of reference $\left(P_{\text {adjusted }}<0.00001\right)$ and an increased emotional reactivity in response to routine social interactions $\left(P_{\text {adjusted }}<0.05\right)$.

There was no difference either in the potential prodrome or in the schizophrenia group, between participants with current depressive disorder and those without on any SPI-A sub-scale (Fig. 2). No significant difference between participants with schizophrenia and depression and those suffering from a potential prodrome and depression showed, however, participants wtih schizophrenia and additional depression exhibited highly significant higher mean totals than participants with depression alone on all SPI-A sub-scales (all $P_{\text {adjust }} \leqslant 0.014$; Fig. 2). Furthermore, 
Table 2 Classification results in groups of stepwise logistic regression equations generated with (regl) or without (reg2) consideration of current depressive disorders

\begin{tabular}{|c|c|c|c|c|}
\hline \multirow[b]{2}{*}{ Participants with: } & \multicolumn{2}{|c|}{ regl classification } & \multicolumn{2}{|c|}{ reg2 classification } \\
\hline & $\begin{array}{c}\text { Depression } \\
n(\%)\end{array}$ & $\begin{array}{c}\text { Prodrome } \\
n(\%)\end{array}$ & $\begin{array}{c}\text { Depression } \\
n(\%)\end{array}$ & $\begin{array}{c}\text { Prodrome } \\
n(\%)\end{array}$ \\
\hline Unipolar depression (MG, $n=59$ ) & $53(89.9)$ & $6(10.1)$ & $48(81.4)$ & II (18.6) \\
\hline Potential prodrome (MG; $n=73$ ) & $8(I I .0)$ & $65(89.0)$ & $15(20.5)$ & $58(79.5)$ \\
\hline Unipolar depression (MV, $n=56$ ) & $53(94.6)$ & $3(5.4)$ & $47(83.9)$ & $9(16.1)$ \\
\hline Potential prodrome (MV; $n=73$ ) & $5(6.8)$ & $68(93.2)$ & $12(16.4)$ & $61(83.6)$ \\
\hline Potential prodrome with depression $(n=56)$ & $13(23.2)$ & $43(76.8)$ & $10(17.9)$ & $46(82.1)$ \\
\hline First-episode schizophrenia $(n=153)^{\prime}$ & $6(3.9)$ & $147(96.1)$ & $33(21.6)$ & $120(78.4)$ \\
\hline $\begin{array}{l}\text { First-episode schizophrenia } \\
\text { with depression }(n=32)^{\prime}\end{array}$ & $6(18.8)$ & $26(81.3)$ & $8(25.0)$ & $24(75.0)$ \\
\hline
\end{tabular}

MG, model generation group; MV, model validation group.

I. Classification of participants with schizophrenia as prodromal was considered correct classification.

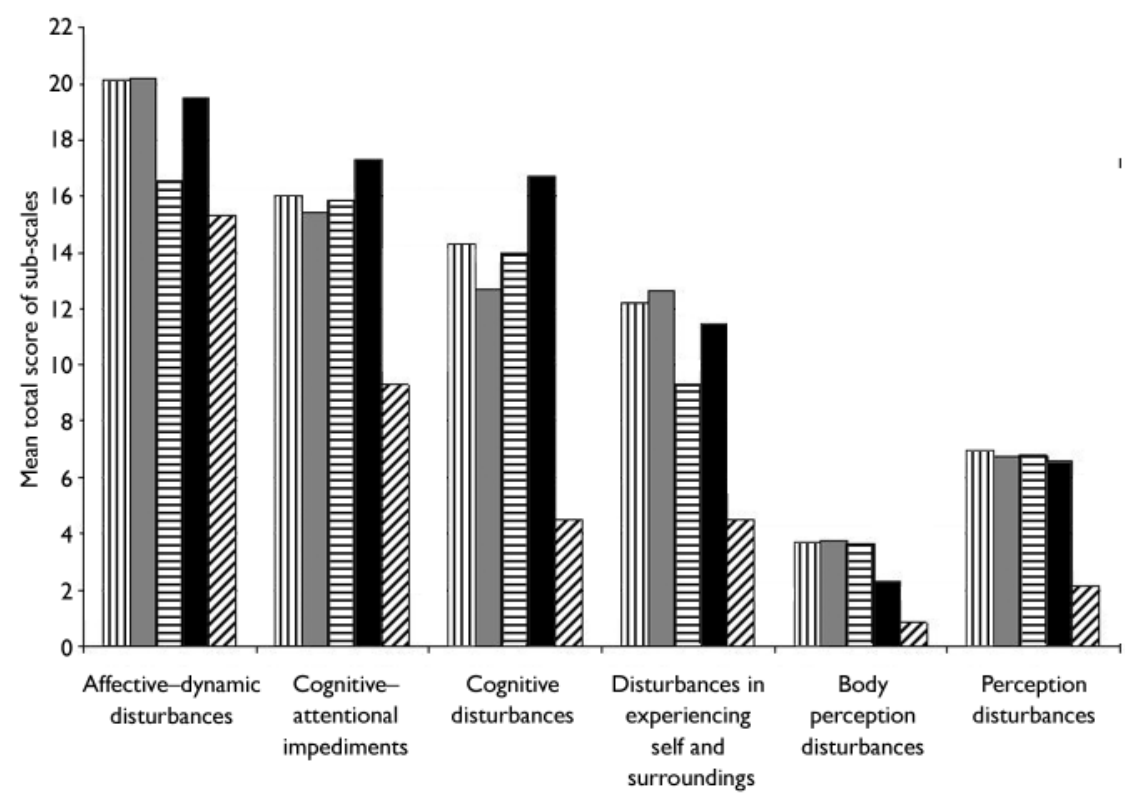

Fig. 2 Group means of the SPI-A sub-scale totals in subgroups. ||[|, schizophrenia, no depression $(n=\mid 2 I)$;

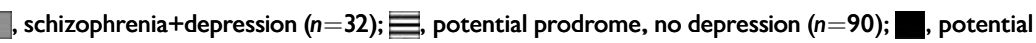

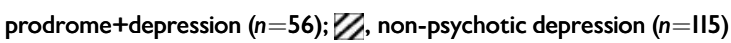

participants suffering from a potential prodrome and depression as well as those not additionally suffering from depression had highly significant higher means on each SPI-A sub-scale $\left(P_{\text {adjusted }} \leqslant 0.0056\right)$, except on affective-dynamic disturbances that did not differ between participants suffering from a potential prodrome but not depression and those with depression alone $\left(P_{\text {adjusted }}=0.179\right)$. Here, only the impaired tolerance to certain everyday stressors was more severe in participants suffering from a potential prodrome but not depression $\left(P_{\text {adjusted }}<0.05\right)$; all the other four items al, 1993; Häfner et al, 1999). Furthermore, depression according to DSM-IV criteria (i.e. major depressive, dysthymic or depressive disorder not otherwise specified) - at $28 \%$ point prevalence - occurred frequently in individuals at ultra-high risk of psychosis (Rosen et al, 2006; Simon et al, 2006). In line with these findings, in our study, $38 \%$ of the participants fulfilling psychopathological research criteria of an early or late initial prodromal state (Ruhrmann et al, 2003) and $21 \%$ of those with first-episode schizophrenia met criteria for a current depressive disorder according to DSM-IV. This emphasises the need to develop criteria for an early detection of psychosis that, among other properties, clearly discriminate between individuals with potential prodrome and those with depression.

Conceptualised originally as primary psychopathological disturbances underlying psychotic symptoms in schizophrenia (Huber \& Gross, 1989; Gross \& Huber, 2005), basic symptoms have already been studied in affective disorders. These studies found that especially the cognitive-perceptive disturbances distinguish schizophrenia from non-psychotic affective disorder (Klosterkötter et al, 1996; Bechdolf et al, 2002) and possibly even psychotic bipolar illness (Parnas et al, 2003). Furthermore, basic symptoms, especially self-experienced cognitive disturbances, have been shown to be highly promising predictors of subsequent first-episode psychosis (Klosterkötter et al, 2001; Lam et al, 2004; Yung et al, 2005) and have reportedly aggregated significantly in schizophrenia (Parnas et al, 2003). These cognitive disturbances, in addition, support a more sensitive and narrow definition of a homogeneous group of atrisk subjects compared to other approaches (Simon et al, 2006). We found comparable expressions of basic symptoms in participants with first-episode schizophrenia and those suffering from a potential prodrome for all sub-scales of the SPI-A, which were decidedly more severe than in non-psychotic depressive subjects, even as regards the more depressive-like complaints subsumed in affective-dynamic disturbances. This gives support to the notion that basic symptoms are indeed a phenomenology specific to the schizophrenia spectrum. This notion is further delineated by the lack of differences within the potential prodrome and schizophrenia groups whether or not they have a diagnosis of a current depressive episode according to 
DSM-IV. Although groups differed significantly in socio-demographic data, the association between socio-demographic data and basic symptoms was negligibly low in correlation analyses, thus not indicating presence of a socio-demographic sample bias.

As in previous studies (Klosterkötter et al, 1996; Bechdolf et al, 2002; Parnas et $a l, 2003)$ and at highly significant adjusted $P$-levels, the differences between the potential prodrome and schizophrenia, respectively, and the depression group were most pronounced in the SPI-A sub-scales cognitive disturbances and disturbances in experiencing self and surroundings. The fact that about half of the items of cognitive disturbances (3 of 6 items) and disturbances in experiencing self and surroundings ( 3 of 5 items) were (at a severity of at least ' 3 ') part of the inclusion criteria of the prodrome is certainly prone to introduce a selection bias into the findings, which might lead to a tautology regarding the comparison of potential prodrome and non-psychotic depressive disorders. While the definition of the putatively prodromal as well as of the participants with nonpsychotic depression was at least partly dependent on SPI-A items, the schizophrenia subjects were a group included completely independently from their performance on the SPI-A. Thus, certain conditions focusing on the performance of these participants with schizophrenia were formulated and tested to estimate the amount to which the results might be influenced by the selection bias. Only three single comparisons did not follow the hypothesised directions: affective-dynamic disturbances and disturbances in self and surroundings were significantly more frequent in participants with schizophrenia but no depression than in those suffering from a potential prodrome but not depression, a result that gives even more support to the view that the high performance of those who are putatively prodromal is not only induced by the inclusion criterion for the prodrome than the hypothesised absence of a group difference. The third comparison that did not show the expected group difference between participants suffering from a nondepressive prodrome and participants with depression alone involved affectivedynamic disturbances was a sub-scale with none of its items being part of the prodromal inclusion criterion. Thus, with only one single comparison truly differing from the initial hypotheses, it seems fair to

FRAUKE SCHULTZE-LUTTER, PhD, MPsych, STEPHAN RUHRMANN, MD, HEINZ PICKER, MPsych, HEINRICH GRAF von REVENTLOW, MPsych, University of Cologne, Department of Psychiatry and Psychotherapy, Early Recognition and Intervention Centre for Mental Crises (FETZ), Cologne, Germany; ANKE BROCKHAUSDUMKE, MD, JOACHIM KLOSTERKÖTTER, MD, University of Cologne, Department of Psychiatry and Psychotherapy, Cologne, Germany

Correspondence: Dr Frauke Schultze-Lutter, University of Cologne, Department of Psychiatry and Psychotherapy, Early Recognition and Intervention Centre for Mental Crises (FETZ), 50924 Cologne, Germany, Tel: +49 221478 6098; fax: +49 221 478 3624; email: frauke.schultze-lutter@uk-koeln.de

deduce that the selection bias had no major impact on the results. However, only follow-up of subjects will conclusively show whether the higher totals of cognitive disturbances and of disturbances in experiencing self and surroundings are truly associated with a conversion to psychosis in the comorbid potential prodrome group.

The prior notion of the importance of cognitive disturbances in the identification of persons symptomatically at an increased risk of psychosis is also supported by the results of the stepwise logistic regression analyses, which at a psychopathological level included mainly cognitive disturbances. This led to high rates of correct classifications of around $80 \%$ in the model validation groups including individuals with schizophrenia. When age and gender as the main socio-demographic variables accounting for group differences were considered along with current depressive disorders, classification rates improved by about a further $10 \%$, thereby selecting current major depressive disorder and current depressive disorder not otherwise specified over age and gender. With a positive impact of psychopathology and a negative impact of current depression, the resulting equation led to a perfect classification of potential prodrome and schizophrenia subgroups without depression and to only few misclassifications of those participants with depression. Yet, the misclassification rates of prodrome and schizophrenia subgroups with current depression were around $20 \%$. Future studies on larger samples and additional assessment of variables that are potentially relevant to depression, such as dysfunctional beliefs, will have to show if the introduction of a second-step classification algorithm especially generated to distinguish depressive subjects with regard to a potential prodrome of psychosis might significantly increase the number of right classifications in this group. Studies on other samples with different (comorbid) psychiatric conditions are needed to decide if such a two-step procedure might be reasonable for other potentially comorbid conditions such as anxiety disorders within the psychotic prodrome.

Thus, basic symptoms, and especially cognitive basic symptoms, seem to be a good tool to distinguish (pre-)psychotic from affective disorders. Furthermore, especially in those individuals suffering from a potential prodrome and a comorbid depressive disorder, a reliable early identification might require a second-step assessment to account for the comorbid condition.

\section{ACKNOWLEDGEMENTS}

The study was supported by a grant from the German Research Foundation (DFG; grant ID KL970/3-I and KL970/3-2) to J.K., F.S.-L. and E.M. Steinmeyer.

\section{REFERENCES}

American Psychiatric Association (1994) Diagnostic and Statistical Manual of Mental Disorders (4th edn) (DSM-IV). APA.

Bechdolf, A., Schultze-Lutter, F. \& Klosterkötter, J. (2002) Self-experienced vulnerability, prodromal symptoms and coping strategies preceding schizophrenic and depressive relapses. European Psychiatry, I7, I-10.

Gross, G. \& Huber, G. (2005) Basic symptoms and prodromal phase of schizophrenia. Neurology, Psychiatry and Brain Research, 12, 185-198.

Gross, G., Huber, G., Klosterkötter, J., et al (1987) Bonner Skala für die Beurteilung von Basissymptomen (BSABS; Bonn Scale for the Assessment of Basic Symptoms). Springer

Häfner, H., Löffler, W., Maurer, K., et al (1999)

Depression, negative symptoms, social stagnation and social decline in the early course of schizophrenia. Acto Psychiatrica Scandinavica, 100, 105-118.

Holm, S. (1979) A simple sequentially rejective multiple test procedure. Scandinavian Journal of Statistics, $\mathbf{6}$, 65-70

Huber, G. \& Gross, G. (1989) The concept of basic symptoms in schizophrenic and schizoaffective psychoses. Recenti Progressi in Medicina, 80, 646-652.

Kay, S. R., Fiszbein, A. \& Opler, L. A. (1987) The Positive and Negative Syndrome Scale (PANSS) for schizophrenia. Schizophrenia Bulletin, I3, 261-276. 
Klosterkötter, J., Ebel, H., Schultze-Lutter, F., et al (1996) Diagnostic validity of basic symptoms. European Archives of Psychiatry and Clinical Neuroscience, 246 147-154.

Klosterkötter, J., Hellmich, M., Steinmeyer, E. M. et al (200I) Diagnosing schizophrenia in the initial prodromal phase. Archives of General Psychiatry, $\mathbf{5 8}$ 158-164.

Koreen, A. R., Samuel, M. D., Siris, S. G., et al (1993) Depression in first-episode schizophrenia. American Journal of Psychiatry, 150, 1643-1648.

Lam, M., Hung, S. F. \& Chen, E.Y. H. (2004) Transition to psychosis in Chinese high risk population. Schizophrenia Research, 70, 21.

Miller, T. J., McGlashan, T. H., Lifshey Rosen, J., et al (2002) Prospective diagnosis of the initial prodrome for schizophrenia based on the Structured Interview for Prodromal Syndromes: preliminary evidence of interrater reliability and predictive validity. American Journal of Psychiatry, 159, 863-865.

Parnas, J., Handest, P., Sæbye, D., et al (2003) Anomalies of subjective experience in schizophrenia and psychotic bipolar disorder. Acta Psychiatrica Scandinavica, 108, 126-133.

Phillips, L. J., Yung, A. R., McGorry, P. D. (2000) Identification of young people at risk of psychosis: validation of Personal Assessment and Crisis Evaluation Clinic intake criteria. Australian and New Zealand Journal of Psychiatry, 34 (suppl.), sl64-sl69.

Rosen, J. L., Miller, T. J., D’Andrea, J.T., et al (2006) Comorbid diagnoses in patients meeting criteria for the schizophrenia prodrome. Schizophrenia Research, in press.

Ruhrmann, S., Schultze-Lutter, F. \& Klosterkötter, J. (2003) Early detection and intervention in the initial prodromal phase of schizophrenia. Pharmacopsychiatry, 36 (suppl.3), 162-167.

Schultze-Lutter, F., Ruhrmann, S., Picker, H., et al (2007) The Schizophrenia Proneness Instrument (SPI-A) - a tool for the assessment of basic symptoms. European Psychiatry, 2l (suppl.I), s27.

Simon, A., Dvorsky, D. N., Boesch, J., et al (2006) Defining subjects at risk for psychosis: A comparison of two approaches. Schizophrenia Research, 8I, 83-90.

Wittchen, H. U., Wunderlich, U., Gruschwitz, S., et al (1997) Strukturiertes Klinisches Interview für DSM-IV. Dt. Bearbeitung. Hogrefe.

Yung, A. R., Pan Yuen, H., McGorry, P. D., et al (2005) Mapping the onset of psychosis: the Comprehensive Asssessment of At-Risk Mental States. Australian and New Zealand Journal of Psychiatry, 39, 964-97I. 